\title{
Applying AI to Cooperating Agricultural Robots
}

\author{
Kurt Nielsen ${ }^{1}$, Jakob Appel ${ }^{1}$, and Yves Demazeau ${ }^{2}$ \\ 1 The Maersk Mc-Kinney Moller Institute for Production Technology, University of \\ Southern Denmark Campusvej 55 DK-5230 Odense M DENMARK \\ \{kurtn, appel\} @ipip. sdu.dk, \\ 2 Laboratoire Leibniz, Institut IMAG 46, avenue Félix Viallet F-38031 Grenoble \\ Cédex FRANCE \\ Yves.Demazeaudimag.fr
}

\begin{abstract}
We consider the experimental development of collaborating robots able to work within fields of crops. Specifically we investigate how AI principles can be applied to this agricultural domain and how the user should be involved in such a system. To support collaboration between agents a representation of responsibilities and dependencies is necessary. This is done by introducing groups and roles, from MAS theory, that the agent must adhere to, formalised by the Agent-GroupRole (AGR) model. To enable a human user to influence the system we adopt the principles declared by the VOWELS paradigm. We then show that the AGR model and the VOWELS paradigm, enable us to solve two practical agricultural problems, and lastly we argue that the obtained results can be transferred to other domains, such as pervasive computing.
\end{abstract}

\section{Introduction}

The AgroBots project[1] deals with various advanced technologies for agriculture, mainly the development of prototypical robots able to establish, maintain, monitor, and harvest fields of crops. In [2] Ferentinos et al. propose a solution for the path planning problem of agricultural robots and in [3] Han et al. propose how agricultural robots can be guided by a vision system. In this paper we investigate how we can obtain collaboration between the robots, by focusing on the "Explorer-Transporter Paradigm", introduced by the authors in [4]. Essentially this paradigm consists of two types of robots, namely explorers who traverse the field(s), exploring weed and crop type and collaborate to build up a precise map of the field and transporters who are holders of different types of fertiliser or pesticides and spray the field(s) based on the information gathered from the explorers. Furthermore, the farmer must be able to intervene with the robots, such that they at all time behave according to his needs and desires.

The remainder of this section is structured as follows: Subsection 1.1 introduces MAS, which were chosen as the analytical approach, as it, to a high degree, captures the characteristics of intelligent robots in an agricultural domain. Subsection 1.2 introduces two practical agricultural problems that we 
have chosen to focus on in this paper. Subsection 1.3 describes the structure of the remainder of the paper.

\subsection{MAS}

In AI research biology, human behaviour and mind are often used as sources of inspiration, and they all serve as the ultimate, but so far unreachable, abstraction for researchers. MAS(MAS) are seen as an abstraction, resembling biology and human behaviour, where an agent is an autonomous proactive entity, that collaborates with its fellow agents and the environment, in order to reach its goals. MAS encapsulate and capture the agents' flexible, autonomous and problem-solving behaviour, the richness of agents interactions, and the organisational structures and social capabilities into one entity. This powerful abstraction is a promising, but so far, a research oriented approach for developing a range of complex, typically, distributed computer systems. In [5] Jennings et al. argue, among others, that MAS provide the ability to solve problems in a natural and efficient way in domains characterised by unpredictability, complexity and distribution. So far, this is more based on assumptions than on practical experiences.

\subsection{Two Agricultural Problems}

There is a great amount of design challenges in connection with the ExplorerTransporter paradigm and consequently we need to narrow down which problems we will focus on. In collaboration with industrial partners[6], we have identified the two following problems that are of both research and corporative interest: (i) Tool Changing: an agricultural robot should be much like a tractor that is able to dynamically change its tool and adapt to new problem domains, (ii) Human Intervention: the user should be able to change and influence the system according to his current and future needs.

\subsection{Structure of the Paper}

Section 2 introduces organisational theory from MAS and adduces how this can help to solve the Tool Changing problem. Section 3 introduces user interaction theory and state the usability of this for the Human Intervention problem. Section 4 summarises the developed system and how the Tool Changing and Human Intervention problems were actually implemented. Section 5 concludes on the work and puts the paper into perspective.

\section{Solving Tool Changing using Organisational Theory}

An agricultural robot should be able to change its working tool dynamically through its lifetime. This entails a change regarding which robots it should and 
can collaborate with, and hence tool changing equals a change of the organisation as will be deduced in the following.

Just as with human organisations, agent organisations provide a description of how the members of the population interact with one another [7]. Agent organisations have properties, norms and authority relationship structures which transcend individual agents and help them to coordinate their actions. The set of properties, norms and structures form the organisational setting, which contains communication lines, authority lines, interactions patterns and descriptions of expected behaviour [8]. In [9], Demazeau makes a more formal description:

$$
\text { Organisation }(M A S)=\text { Roles }+ \text { Links }
$$

that is, the organisation is a set consisting of all the roles that exist in the system, and of all the links among the agents that connect the roles one to another, and show the mutual connectivity between roles. The roles and interactions are typically expected to be relatively stable and change slowly over time [10].

\subsection{The Role of Roles}

If we were to decompose organisations we would at some stage end up in the building block called role [11]. Roles are an inherent part of organisations, and are a way of describing behaviour, responsibility and interaction [12]. The following constraints are general in MAS when an agent takes on a role: (i) Obligations and responsibilities: $\mathbf{A}$ role is inherently connected with certain duties, and a role, therefore, has responsibilities, (ii) Requirements and abilities: Not all agents can take on a role, they often require physical and/or logical abilities.

From the work represented by Masolo et al. in [12] we adopt the following dynamic properties of roles: (i) An agent can play different roles simultaneously, (ii) An agent can change a role, (iii) An agent can play the same role several times simultaneously, meaning that an agent might be acting different specialisations of the same role at a time, (iv) A role can be played by different agents, simultaneously or at different times.

The above properties means that roles can be assigned to agents in at least two ways: endogenously (emergent self-organisation as the system runs) or exogenously (by the designer when the system is initialised) [10]. Note that an agent can play multiple roles, as long as they do not coincide in their responsibility area, which would result in a conflict and a dysfunctional organisation.

In the next subsection we introduce the AGR model, which is an effective tool to model an organisation and to help limit irrelevant information processing.

\subsection{AGR}

In [13] the notion "Organisation Centered MAS" is introduced. It is built on the concept of "groups" and "roles", where groups refer to an overall partitioning of 
the organisation, which may be overlapping. Groups and roles were combined by Ferber and Gutknecht in [14] that proposed an organisational based modelling scheme, called AGR for AGR, to describe MAS in organisational terms. Only agents that are members of the same group may communicate, but agents can be members of several groups simultaneously. Thereby groups can be used to regulate, foster, or support the interaction of those agents within the group [15]. By complying to the AGR model we propose the agent architecture depicted in figure 1. For simplicity we have replaced the representation of the internal

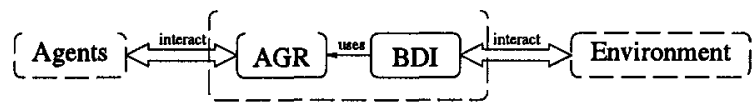

Fig. 1. The agent architecture with the addition of the AGR model.

Belief-Desire-Intention(BDI) architecture with a box called BDI. There has been many approaches in the AI community for an agent architecture, but as BDI is far the most notorious and respected agent model it was selected. For an introduction to BDI see [16] and for a more elaborated explanation of how we specifically implemented the BDI box see [4].

\subsection{Tool Changing Design}

If we decompose the result of a tool change we get the following results: (i) Change of obligations and responsibilities: a tool is a physical representation of a certain capability. Inherently an agent's capability is connected to a number of obligations and responsibilities which the agent should attend to with the certain capability, (ii) Change of mutual dependencies: when changing tool an agent should also change its surroundings expectations towards the agent and its own mutual dependencies.

By implementing an AGR structure, we can model the change of obligations and responsibilities as a change of role. The other result of a tool change, change of mutual dependencies, can be modelled as a change of group, since a group is defined as a clustering of activity dependent and interacting agents. Hence, by letting an agent dynamically change which roles it play and which groups it participate in, it is able to change its tool throughout its lifetime and still maintain the proper collaborative links.

\section{Solving Human Intervention using User Interaction Theory}

So far, decisions that have to be made within the agricultural establishment have, to a high degree, been taken by the individual farmer. Hence, it is highly unlikely that he overnight wants to assign full control to the robots, and not at some point wish to overrule a robots decision or rework its strategies. 
The notion of a user has been remarkably absent in the MAS literature. One of the reasons for this is probably that MAS are still far from having their true breakthrough both research and industry wise and, therefore, the need to represent the user has so far been minimal[17]. In this system, however, we can not ignore the user's perspective, as we see it as an inherent part in any agricultural process, independently of whether the robots are autonomous or controlled directly by a user.

\subsection{The Role of the User}

In almost every commercial software system the user will at some point wish to take control of, or at least alter, the system. In MAS, this can be done by enabling the user to change an agent's beliefs, desires, intentions and capabilities, both via normal agent interactions, but also due to other entry points to the agent, that only exist for the user. This enables the user to modify or replace work processes, both directly by updating the intentions and capabilities, but also by affecting beliefs and desires. In the next subsection we present the VOWELS paradigm, which recognises the user as an essential part of a MAS.

\subsection{The VOWELS Paradigm}

The VOWELS paradigm is a popular decomposition model that breaks down MAS into its constituent parts. The four basic bricks of the VOWELS paradigm, initially introduced by one of the coauthors in [18], are the Agents, the Environment, the Interactions and the Organisations (A, E, I, O). In [19], the lack of a user representation is discussed as the user can hardly be described with the same terms as an agent, which result in the addition of a user(U). In addition to these bricks, the VOWELS paradigm is guided by a declarative principle, which states that a MAS will be composed of agents, environments, interactions, organisations and users:

$$
M A S=A+E+I+O+U
$$

\subsection{Human Intervention Design}

In order to model an agent in a realistic MAS we have to take into account the effects that the user can have upon an agent's beliefs, desires, capabilities, groups and roles. This leads to an extension of the agent architecture diagram, presented in figure 1, which by the addition of the VOWELS gives us the model depicted in figure 2. By enabling the user to modify an agent's role and/or membership of a group, the user is able to e.g. make a tool change and by modifying an agent's capabilities, beliefs and/or desires a new behaviour can be obtained. Thus, by complying to the VOWELS paradigm and acknowledge the user's part in a MAS, we obtain a system that provides the desired flexibility with respect to the intervention of a human user, and thereby we achieve a solution for the Human Intervention problem. In the next section we will explain how we implemented the two agricultural problems. 


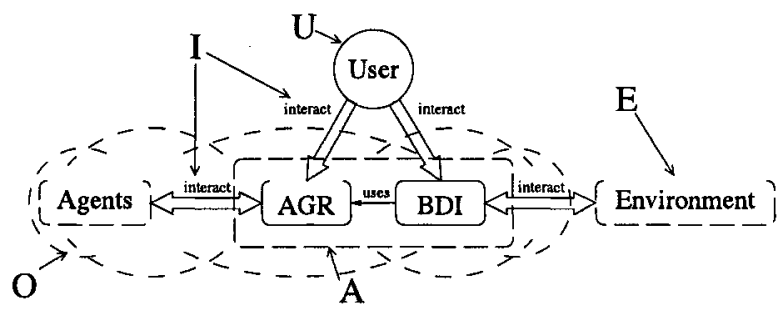

Fig. 2. The agent architecture with the addition of the user. The VOWELS bricks, including the new $U$, are added to show them in a more practical context.

\section{Implementation}

To verify and experiment with the proposed solutions from section 2 and 3 for the Tool Changing and Human Intervention problem, three prototype platforms have been used. The software kernels used are the same on each of the platforms facilitating three different levels of realism: (i) AgroBOT: an advanced robotic platform developed by Bygholm[20], consisting of physical robots that can navigate in an agricultural field, (ii) LEGOBOT: a laboratory set-up developed by the Maersk Institute [21], that apply simple physical robots for simulation, (iii) SoftBOT: a virtual robot platform that is used as an abstraction of the two above listed robots and, therefore, allows for fast developing.

The overall goal of the considered part of the AgroBots project is to develop the AgroBOT platform itself. However, in order to speed up development time and to facilitate experiments with alternative designs of strategies and techniques, the LEGOBOT and SoftBOT platforms were introduced, which is the focus of this paper.

\subsection{The Setup}

The developed system provides an overview window that depicts the field and the agents within it, as visualised in figure $3(\mathrm{a})$. The agents are represented as squares with different colours according to their role and the agents' unique identification numbers are printed upon the squares, so that the user can identify the agents. The LEGOBOTs physically move upon a restricted area, whereas the SoftBOTs are projected upon the restricted area as squares with their unique Id number depicted in the middle. This construction is shown in figure $3(\mathrm{~b})$.

\subsection{Experimenting with Tool Changing and Human Intervention}

The AGR model has been implemented providing the agents with the organisational properties listed in section 2. Furthermore, the user is able to interact with the agents, through interaction agents(IA), both as an observer that is 

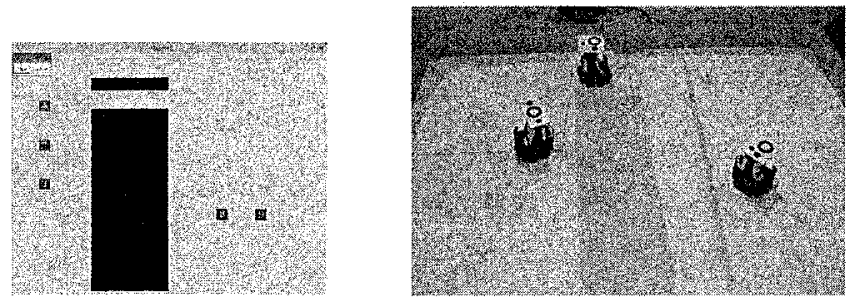

(a) The LEGOBOTs and the Soft- (b) The LEGOBOTs and the SoftBOTs BOTs in the overview window. in their physical environment.

Fig. 3. The virtual and physical environment, both containing black box-shaped obstacles.

able to monitor the agent's internal state, but also as an active player that can change the agent's roles and exchange plans dynamically. Plans are an inherently part of the BDI model, introduced in subsection 2.2 , and can be thought of as a recipe for achieving a goal.

The interaction agents are activated by clicking on an agent icon on the overview window and are so far able to do the following: (i) Show roles: the IA is capable of showing all roles that an agent has the potential to be. That is both its active and none-active roles, (ii) Activ ate and deactivate role: the IA activates and deactivates roles, (iii) Show plans: the IA shows the current plans that an agent currently possesses, (iv) Exchange plan: the IA dynamically exchanges plans to another agent.

\subsection{Last Words Regarding the System}

The developed system uses concepts from MAS frameworks such as JACK[22] (capabilities and plans) and MadKit[23] (AGR model), but also concepts which, to our knowledge, can not be found in any MAS framework today such as dynamic exchange of plans and direct user interaction with agents' mutual dependencies and responsibilities, which makes the system unique in MAS context.

\section{Conclusion}

In this paper we have advocated the applicability of group-based role definitions for intelligent agricultural robots. Furthermore a representation of an user was introduced as an indispensable part of an agricultural system and MAS in general. To substantiate these claims two real world agricultural problems were implemented, namely Tool Changing and Human Intervention. This proved the AGR model to not only be of theoretical interest and that user interaction is of vital importance within AI applications. Allowing agents to change their roles and group memberships dyaamically gives the agents the opportunity to adapt to changes in their environment, or even to change their application domain, 
whereby the system is not only applicable for agricultural robots or alike, but also within areas with other characteristics.

Most computer researchers agree that computer systems are going to be everywhere and will always be connected and active, referred to as pervasive computer systems. The MAS approach has relevance to pervasive computing as they both rely on situatedness, openness, locality in control and locality in interactions. However, the MAS approach suffers from the absence of the human input and interaction, which is a core aspect of pervasive computing. We have proposed the VOWELS paradigm as a start and we believe that the aspect of human interaction with MAS will become a major research area in the future.

\section{References}

1. http://www.agrobots.dk.

2. Ferentinos, K.P., Arvanitis, K.G., Sigrimis, N.: Heuristic optimization methods for motion planning of autonomous agricultural vehicles. J. of Global Optimization 23 (2002) 155-170

3. Han, S., Zhang, Q., Ni, B., Reid, J.: A guidance directrix approach to visionbased vehicle guidance systems. In: COMPUTERS AND ELECTRONICS IN AGRICULTURE 43 (3). (2004) 179-195

4. Appel, J., Nielsen, K.: Applied multi-agent systems principles to cooperating robots in tomorrow's agricultural environment. Master's thesis, University of Southern Denmark Campusvej 55 DK-5230 Odense M DENMARK (2005)

5. Jennings, N.R., Wooldridge, M.: Applying agent technology. Applied Artificial Intelligence 9 (1995) 351-361

6. http://www.agrobots.dk/deltagere.htm.

7. Horling, B., Lesser, V.: A Survey of Multi-Agent Organizational Paradigms. Computer Science Technical Report 04-45, University of Massachusetts (2004)

8. Karageorgos, A., Mehandjiev, N., Thompson, S.: Ramasd: a semi-automatic method for designing agent organisations. Knowl. Eng. Rev. 17 (2002) 331-358

9. Demazeau, Y., Costa, A.C.R.: Populations and organizations in open multi-agent systems (1996)

10. Odell, J., Parunak, H., Fleischer, M.: The role of roles in designing effective agent organizations (2003)

11. Gasser, L.: Perspectives on organizations in multi-agens systems (2001)

12. Masolo, C., Vieu, L., Bottazzi, E., Catenacci, C., Ferrario, R., Gangemi, A., Guarino, N.: Social roles and their descriptions (2004)

13. Ferber, J., Gutknecht, O., Michel, F.: From agents to organizations: An organizational view of multi-agent systems. In Paolo Giorgini, Jrg P. Mler, J.O., ed.: Agent-Oriented Software Engineering IV. Springer-Verlag (2003) 214-230

14. Ferber, J., Gutknecht, O.: A meta-model for the analysis and design of organizations in multi-agent systems (1998)

15. Odell, J., Nodine, M., Levy, R.: A metamodel for agents, roles, and groups (2005)

16. Nagi, K.: Transactional Agents. Springer-Verlag (2001)

17. Albert, P., Demazeau, Y., Elfallah-Seghrouchni, A.: Incitations et freins à la mise en oeuvre de sma. In: Systèmes Multi-Agents. (2004) 261-289 (In France). 
18. Demazeau, Y.: From interactions to collective behaviour in agent-based systems (1995)

19. Demazeau, Y.: Créativité émergente centrée utilisateur. In: 1lèmes Journées Francophones sur les Systèmes Multi-Agents. (2003) 31-36 (In France).

20. The Danish Agricultural Research Centre of Horsens, http://www.agrsci.dk/jbt.

21. The Maersk Mc-Kinney Moller Institute for Production Technology, University of Southern Denmark, http://www.mip.sdu.dk/.

22. http://www.agent-software.com/.

23. http://www.madkit.org/. 